\title{
Role of Gut Microbiota Dysbiosis in Breast Cancer and Novel Approaches in Prevention, Diagnosis, and Treatment
}

Sheila W. Ruo ${ }^{1}$, Tasnim Alkayyali ${ }^{2,3}{ }^{3}$, Myat Win ${ }^{4,1}$, Anjli Tara ${ }^{1,5}$, Christine Joseph ${ }^{6}$, Amudhan Kannan 7,1 , Kosha Srivastava ${ }^{8}$, Olive Ochuba ${ }^{9}$, Jasmine K. Sandhu ${ }^{10}$, Terry R. Went ${ }^{11}$, Waleed Sultan ${ }^{12,11,13}$, Ketan Kantamaneni ${ }^{14,15}$, Sujan Poudel ${ }^{16,17}$

1. General Surgery, California Institute of Behavioral Neurosciences \& Psychology, Fairfield, USA 2. Internal Medicine, Marmara University, Istanbul, TUR 3. Pathology, California Institute of Behavioral Neurosciences \& Psychology, Fairfield, USA 4. General Surgery, Nottingham University Hospitals NHS Trust, Nottingham, GBR 5. General Surgery, Liaquat University of Medical and Health Sciences, Jamshoro, PAK 6. Urology and Obstetrics \& Gynecology, California Institute of Behavioral Neurosciences \& Psychology, Fairfield, USA 7. General Surgery, Jawaharlal Institute of Postgraduate Medical Education and Research, Puducherry, IND 8. Neurology, California Institute of Behavioral Neurosciences \& Psychology, Fairfield, USA 9. Internal Medicine, California Institute of Behavioral Neurosciences \& Psychology, Fairfield, USA 10. Obstetrics \& Gynecology, California Institute of Behavioral Neurosciences \& Psychology, Fairfield, USA 11. Medicine, California Institute of Behavioral Neurosciences \& Psychology, Fairfield, USA 12. Medicine, Beni Suef University Faculty of Medicine, Beni Suef, EGY 13. Surgery, Halifax Health Medical Center, Daytona Beach, USA 14. Surgery, California Institute of Behavioral Neurosciences \& Psychology, Fairfield, USA 15. Surgery, Dr.Pinnamaneni Siddhartha Institute of Medical Sciences and Research Foundation, Gannavaram, IND 16. Psychiatry and Behavioral Sciences, California Institute of Behavioral Neurosciences \& Psychology, Fairfield, USA 17. Division of Research \& Academic Affairs, Larkin Community Hospital, South Miami, USA

Corresponding author: Sheila W. Ruo, sheilakarey@gmail.com

Received 07/24/2021 Review began 08/11/2021 Review ended 08/19/2021 Published 08/26/2021

\section{(c) Copyright 2021}

Ruo et al. This is an open access article distributed under the terms of the Creative Commons Attribution License CC-BY 4.0., which permits unrestricted use, distribution, and reproduction in any medium, provided the original author and source are credited.

\begin{abstract}
Breast cancer is the most common cause of cancer-related deaths in women. Breast cancer is still a major cause of morbidity and mortality among women despite all the available diagnostic and treatment modalities. The gut microbiota has drawn keen interest as an additional environmental risk factor in breast cancer, especially in sporadic cases. This article explores factors that disrupt the normal gut microbial composition and the role of gut microbial dysbiosis in the development of breast cancer. We finalized 40 relevant articles after searching Pubmed and Google Scholar using regular keywords and the Medical Subject Headings (MeSH) strategy. Gut microbiota dysbiosis has been shown to play a role in the development of breast cancer via estrogen-dependent mechanisms and non-estrogen-dependent mechanisms involving the production of microbial-derived metabolites, immune regulation, and effects on DNA. The gut microbiota influence estrogen metabolism hence estrogen levels. The metabolites that have demonstrated anticancer properties include lithocholic acid, butyrate, and cadaverine. New approaches targeting the gut microbiota have come up and may yield new advances in the prevention, diagnosis, and treatment of breast cancer. They include the use of prebiotics, probiotics, and hormone supplements to restore normobiosis in the prevention and treatment of breast cancer.
\end{abstract}

Categories: General Surgery, Oncology, Nutrition

Keywords: oncology, gut microbiota, dysbiosis, estrobolome, breast cancer, intestinal microbiome

\section{Introduction And Background}

The most common cancer in women, excluding skin cancers, is breast cancer. In 2020, female breast cancer was found to be the cancer with the highest incidence, with an estimated 2.3 million cases (11.7\% of total cancer cases) surpassing lung cancer (11.4\% of total cancer cases) for the first time [1]. It is currently the most frequent cause of cancer death in women worldwide, with about 685,000 estimated deaths in 2020 [1]. About one in eight women in the United States (US) will develop breast cancer in their lifetime [2]. The predicted worldwide incidence of female breast cancer by 2050 is estimated to be 3.2 million new cases per year [2]. These alarming numbers reflect the need for strict screening measures, prevention and treatment strategies, and further studies to understand all possible factors influencing the development of breast cancer. Incidence rates of breast cancer have been rising by about $0.5 \%$ per year, partially due to continued decline in the fertility rate and increased body weight [3].

Breast cancer risk factors have been widely known and discussed over the years [4]. A small subgroup (5$10 \%$ ) of breast cancer cases have been linked to inherited gene mutations [5]. The concept of hormonedriven breast cancer is known, and appropriate treatment modalities are directed towards hormone receptor-positive cancers. Increased breast density on mammography and first-degree relatives with breast cancer were both associated with at least a two-fold increase in breast cancer risk in women aged 40-49 years [6]. Unfortunately, the etiology of sporadic breast cancer cases is not well known. Therefore, it is important to investigate factors involved in the development of sporadic cases, in order to develop appropriate prevention strategies. 
Microbiota refers to the entirety of microbes, both commensals and potentially pathogenic microbes (bacteria, archaea, fungi, viruses, and protozoa), in a particular habitat $[7,8]$. In this article, the word microbiota has been used primarily to refer to bacteria. Microbiome refers to the collection of genomes of microbiota and is commonly used to describe the entity of microbial traits or functions encoded by the microbiota [7]. The terms 'microbiota' and 'microbiome' are now often used synonymously $[7,8]$.

Dysbiosis refers to an abnormal composition of the microbiome in the entire body or a body compartment [8]. There is evident variability of the microbiome between organs and from one individual to the other, as depicted in the Human Microbiome Project (2008 to 2013), making it a potential determinant of disease. The composition of the human microbiota has also been implicated in the development and aggressiveness of cancers [8]. The largest proportion of the human microbiota resides in the gut.

Bacterial communities within a host could be one additional environmental factor related to breast cancer that has only been recently considered in sporadic breast cancers of unknown etiology [8]. Recently, efforts have been made towards fully characterizing the microbiota in different body parts under different health conditions, including breast cancer. Bacterial microorganisms interact with the host metabolic processes and can impact homeostasis. The most relevant strategies now used in microbiota research include 16S rRNA gene next-generation sequencing (NGS), a targeted approach, and a large-scale metagenomics approach, also known as shotgun sequencing [8]. Both quantitative and qualitative differences between gut microbiota in healthy people and people with breast cancer have been explored in a few studies, but a conclusive disease-causing microbial profile has not been determined.

This article aims to elaborate on the role of the disruption of the gut microbiome (dysbiosis) in breast cancer development via estrogen-dependent and non-estrogen-dependent mechanisms [9]. We discuss the causes of microbial dysbiosis and how its prevention or correction can reduce the risk of sporadic breast cancers. Another aim of this article is to shed some light on the new possibilities of involving interventions targeting the intestinal microbiome in diagnosing and treating breast cancer. This article also explores the relevance of the identification of the gut microbiome and its metabolites in stratifying patients for the risk of breast cancer. Potential treatment strategies for breast cancer targeting the gut microbiome, such as prebiotics, probiotics, and dietary interventions, are still in their infancy and are explored further in this review.

\section{Review}

\section{Method}

We performed a search on PubMed and Google Scholar using regular keywords and using the MeSH strategy; 'Breast microbiome' OR 'microbiota' AND 'gastrointestinal' OR 'gut' OR 'fecal microbiome' AND 'dysbiosis' AND 'breast cancer' (Table 1). We included articles with age groups above 19 years, studies with female subjects, and the English language. We excluded articles and trials not related to gut microbial dysbiosis and the role of gut microbiota in breast cancer in general.

\begin{tabular}{|c|c|c|c|}
\hline Search strategy & $\begin{array}{l}\text { Database } \\
\text { used }\end{array}$ & $\begin{array}{l}\text { Number of articles } \\
\text { before customization } \\
\text { and screening }\end{array}$ & $\begin{array}{l}\text { Number ot articles } \\
\text { after customization } \\
\text { and screening }\end{array}$ \\
\hline Breast Microbıome OR Microbıota & Pubmed & 651 & 33 \\
\hline GastroIntestınal OR Gut OR Microbıome OR Microbıota & Pubmed & $15 / 2$ & 19 \\
\hline recal microbıome OR microbıota in Breast cancer & Pubmed & 155 & 45 \\
\hline Intestinal Dysbiosis in Breast cancer & Pubmed & 6 & 6. \\
\hline Dysbiosis in Breast cancer & Pubmed & 27 & 22 \\
\hline Breast Cancer & Pubmed & 122,048 & - \\
\hline Fecal Microbiome OR Microbiota AND Dysbiosis AND Breast Cancer & Pubmed & 44 & 44 \\
\hline Gut microbiota dysbiosis in Breast Cancer. & $\begin{array}{l}\text { Google } \\
\text { Scholar }\end{array}$ & 5 & 5 \\
\hline
\end{tabular}

TABLE 1: Table showing keywords and search strategy used in the database search

\section{Results}


We screened the articles by title and by the content of the abstracts and full texts. The total number of articles after the initial screening process was 174. Duplicates were removed leaving 87 articles. After further screening by content, the final number of articles used for this paper was 43 , comprising two clinical trials, one systematic review and meta-analysis, 17 review articles, 19 observational studies, three animal studies, and an in vitro study.

\section{Discussion}

Factors influencing gut microbiota composition

The main phyla of the gut microbiota are Firmicutes, Bacteriodetes, Actinobacteria, Proteobacteria, Fusobacteria, Verrucomicrobia, Tenericutes, and Lentisphaerae [10]. The main genera are Bacteroides, Clostridium, Faecalibacterium, Eubacterium, Ruminococcus, Peptococcus, Peptostreptococcus, Lactobacillus, Streptococcus, Streptomyces, and Bifidobacterium [10]. The gut microbiota helps in digestion, metabolism, and immune response, giving rise to a symbiotic relationship between the host's gut and the microbiota called normobiosis, which maintains homeostasis [10]. Dysbiosis disrupts the normal gut-microbiota interaction [10]. It occurs when harmful bacteria outcompete beneficial bacteria (commensals) and/or a reduction in alpha diversity which can lead to disease [10]. Factors influencing the gut microbiota composition include host factors like age, ethnicity, hormonal levels, and environmental factors such as diet, prebiotics, probiotics, stress, hygiene, alcohol, smoking, antibiotic use, chemotherapy, and radiation (Figure 1) [11]. The most explored factors are diet, antibiotics, prebiotics, and probiotics and they are briefly described below.

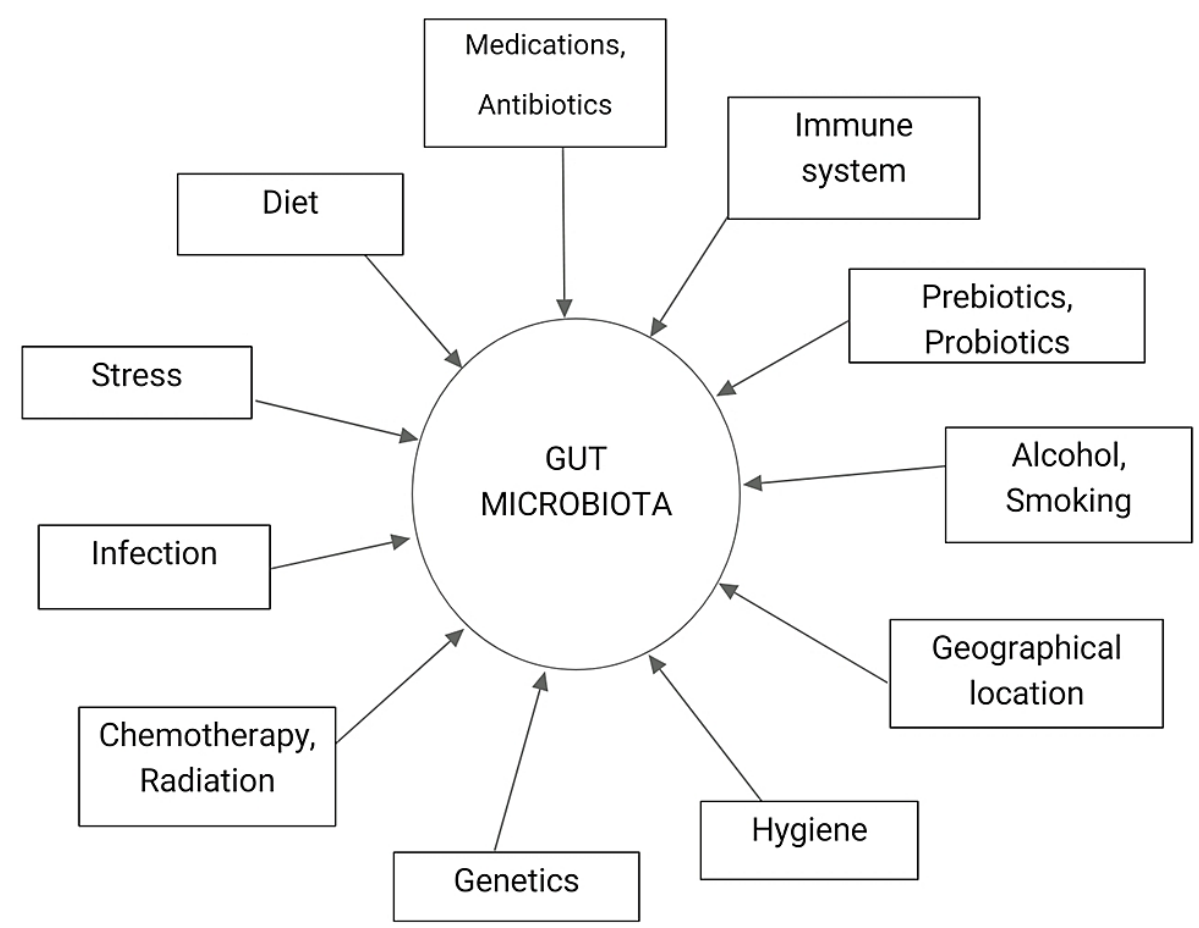

FIGURE 1: Factors that influence gut microbiota composition causing dysbiosis.

Diet

Distinct patterns of intestinal microbiota composition are associated with habitual consumption of animal fats, dietary fiber, and vegetables [12]. High-fiber diet influences gut microbiota composition and lower gut bacterial beta-glucuronidase activity [10]. This reduces deconjugation and reabsorption of estrogens, increasing the fecal excretion of estrogen compounds, leading to a reduction in estrogen levels. Vegetarian women had an increased fecal output leading to a three times increase in fecal excretion of estrogen, reduced fecal glucuronidase activity ( $\mathrm{p}<05$ ), and a resultant $15-18 \%$ decrease in plasma estrogen levels when compared with non-vegetarian women $[13,14]$. Immigrant women on a low-fat diet (20\%-25\% of calories) had lower systemic estrogen levels than American women eating a high-fat diet (40\% of calories) by 30\% [13]. Further correlation in the study, showed that plasma estrogen levels were directly associated with fat and inversely associated with fiber [13]. The Western-type diet increases sex hormone levels and decreases the sex hormone-binding globulin increasing steroid hormone availability for tissues like the breast, giving a sex 
hormone pattern that is commonly seen in breast cancer [15]. Newman et al. reviewed the antineoplastic molecular mechanisms of the Mediterranean diet on the gut microbiome and concluded that the Mediterranean diet could be beneficial in the prevention and treatment of breast cancer [12]. An association between diet and estrogen metabolism has been noted. Dietary fiber can induce bacterial enzyme selectivity by influencing the microbial composition, although other factors may contribute [10]. It also influences bile acid metabolism by interrupting the enterohepatic circulation [10]. Thus, the diet directly affects the gut microbiota composition, estrogen levels, and production of microbial-derived metabolites, which may impact the risk of breast cancer.

Antibiotics, Prebiotics, and Probiotics

Antibiotics usage can alter the gut microbiota composition depending on the class of antibiotic, dose, period of exposure, and target bacteria from the mode of action [10]. Some studies showed that antibiotic consumption (especially prolonged use) was associated with an increased risk of breast cancer development and recurrence, possibly by reducing the diversity of gut microbiota, with one of the studies, describing only a weak correlation [16-18]. In contrast, two studies by Sorensen et al. and Garcia Rodriguez et al. found no increase in the risk of breast cancer with antibiotic use [19,20]. Given the inconclusive evidence, a causeand-effect relationship cannot be established, and some uncontrolled confounding factors may be involved, such as hormone-related diseases that the antibiotics were used for, like acne [18]. Despite the conflicting studies, there is still a cause for prudent use of antibiotics, especially for long-term purposes. Further studies are needed to establish whether an association exists between antibiotic use and breast cancer and this would strengthen the hypothesized relationship between gut microbiota perturbation and breast cancer.

On the other hand, prebiotics and probiotics help maintain proper microbial composition and restore normal balance. Prebiotics such as enterolactone is typically indigestible fiber compounds that enhance the growth and activity of beneficial gut microorganisms. Some, such as phytoestrogens, work as antioxidants causing downregulation of cyclooxygenase-2 (COX-2) mediated inflammation and are shown to be beneficial in cancer prevention [10]. Probiotics are living microorganisms that, when administered in adequate amounts, confer a health benefit to the host [10]. Consumption of probiotics containing live bacteria such as Lactobacillus spp. causes changes in the intestinal microbiome composition and has been shown to decrease the activity of fecal beta-glucuronidase [21,22]. This reflects a decrease in estrogen levels and hence a decrease in the risk of breast cancer.

\section{The crosstalk between the gut microbiota and breast cancer}

Breast cancer development is influenced by estrogen-dependent and non-estrogen-dependent functions of the gastrointestinal microbiome involving the production of bioactive metabolites $[10,23]$. Intestinal microbiota dysbiosis has been linked to breast cancer because certain gut bacteria alter the production of the beneficial anticancer metabolites and disrupt estrogen metabolism in the gut.

Estrogen Dependent Effects: Gut Microbiota-Estrobolome-Breast Cancer Connection

The risk of estrogen-driven breast cancer in postmenopausal women is associated with the concentration of circulating estrogens and duration of exposure to estrogen [24]. The estrobolome refers to the aggregate of intestinal bacterial genes whose products metabolize estrogen, hence may influence the risk of estrogen receptor-positive breast cancer in postmenopausal women [25]. Estrogens are conjugated in the liver and released into bile, which then enters the intestine. Some intestinal bacteria secrete beta-glucuronidase, which deconjugates estrogens that were marked for fecal excretion allowing binding to their receptors [26]. Ervin et al. showed that human gut microbial beta-glucuronidase enzymes could reactivate two estrogen glucuronides, estrone-3-glucuronide and estradiol-17-glucuronide, to estrone and estradiol [27]. However, inhibition of this estrogen reactivation did not prevent tumor development in the mouse model of breast cancer, meaning there are many other processes involved [27]. The free estrogens are reabsorbed via the enterohepatic circulation and reach distant tissues like the breast (Figure 2) [8]. In addition, gut microbes break down indigestible dietary polyphenols to biologically active estrogen-like metabolites with varying estrogenic potency. Gut bacteria also induce the synthesis of estrogen inducible growth factors such as estromedin, which can be involved in carcinogenesis $[8,28]$. Some commensals have the capability via sulfatase to convert inactive steroids into active estrogen [24]. Activation of estrogen receptors in the breast increases the number of cells entering the G0 and G1 phases, stimulating cell proliferation, which is a common finding in breast cancer [29]. Activation of the estrogen receptors in mitochondria induces mitochondrial oxidation, resulting in increased mitochondrial production of free radicals, promoting breast cancer development [23]. 


\section{Cureus}

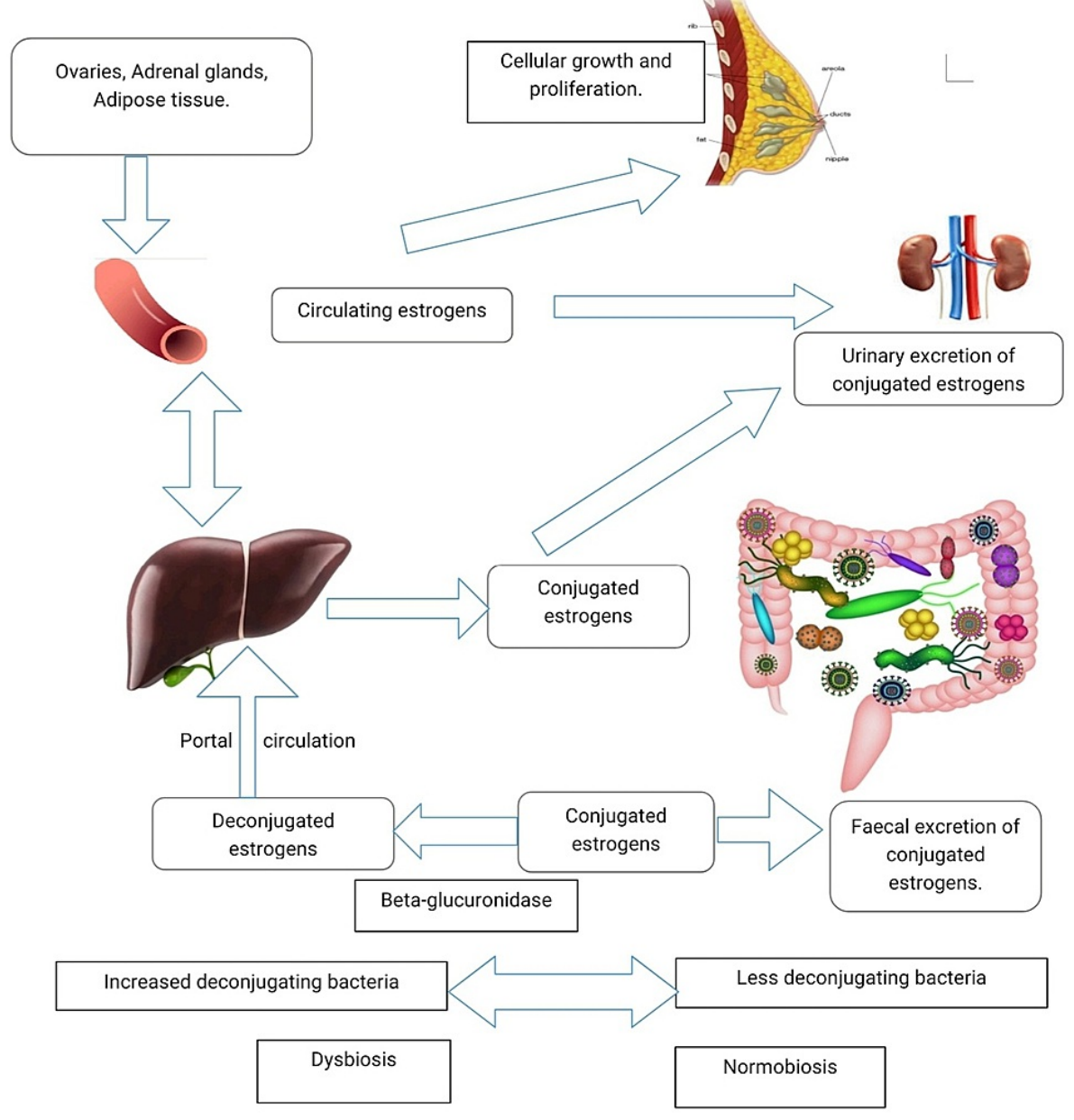

\section{FIGURE 2: The effect of the gut microbiota on the metabolism and enterohepatic cycling of estrogen}

Adapted from: Kwa M., et al., Natl Cancer Inst, 2016 [11].

The proportion of beta-glucuronidase-producing gut bacteria influences the levels of estrogens and their potential breast cancer-associated effects. Disproportionally high levels of such bacteria may be present during dysbiosis. Bacterial beta-glucuronidases are encoded by GUS genes found in most gut bacteria, most commonly Firmicutes, and $B G$ genes present in Firmicutes and Bacteroidetes [23]. Beta-glucuronidaseproducing bacteria include Bacteroides, Bifidobacterium, Clostridium, Citrobacter, Escherichia,

Faecalibacterium, and Propionibacterium, to name a few [30,31]. Therefore, beta-glucuronidase deconjugation activity is dependent on gut microbial composition and diet [26]. Bard et al. found that absolute numbers of Bifidobacterium and Blautia varied significantly according to the clinical stages of breast cancer patients, and other bacterial counts varied with BMI [31].

The effect of the gut microbiome diversity on the levels of estrogens and their metabolites has been explored in various studies. Fuhrman et al. found that healthy postmenopausal women with increased gut microbiome diversity and an abundance of four Clostridia taxa had an increased urinary ratio of hydroxylated estrogen metabolites to parent estrogens, which is related to the etiology of breast cancer [32]. Similarly, Flores et al. showed that total urinary estrogen levels were directly associated with fecal microbiome richness, alpha diversity ( $\mathrm{R} \geqslant 0.50, \mathrm{P} \leqslant 0.003)$, and Clostridia taxa in healthy postmenopausal women and men but not in premenopausal women [33]. Fecal beta-glucuronidase activity correlated significantly with estrone levels and inversely with total estrogens in older adults [33]. This suggests that the influence of gut microbiota on estrogen-driven diseases is limited to older women and men but is absent in premenopausal women. Likewise, a metagenomic analysis demonstrated differences in the functions and composition of gut microbiota amongst the subjects [34]. Postmenopausal breast cancer patients had a different relative abundance of gut microbiota and higher microbial diversity compared to controls [34]. However, no remarkable discrepancy was found between premenopausal cases and controls [34]. In contrast, Goedert et al. concluded that postmenopausal women with breast cancer had altered gut microbiota 


\section{Cureus}

composition but with estrogen-independent low alpha diversity $(\mathrm{p}=0.04)$ [35]. The contrast in these findings on levels of gut microbiota diversity and breast cancer could be because the microbiota can also be affected by the stage and outcomes of the disease [10]. In that regard, Miko et al. observed that the most dramatic decrease in microbial diversity was observed in the early stages of breast cancer [36]. These studies imply that variations in gut microbial diversity amongst women result in differences in estrogen levels,

influencing breast cancer development. Further investigation of the correlation between specific microbiota profiles, for example, Clostridia taxa and estrogen levels, can identify potential targets for intervention [33]. As demonstrated in these studies, the gut microbiome is an important determinant of estrogen metabolism in postmenopausal women (Table 2). 


\section{Cureus}

\begin{tabular}{|c|c|c|c|c|c|c|c|}
\hline Author & Year & Study type & No. & Age & Purpose & Results & Conclusion \\
\hline $\begin{array}{l}\text { Bard JM } \\
\text { et al. } \\
\text { [31] }\end{array}$ & 2015 & $\begin{array}{l}\text { Observational } \\
\text { study: Cross- } \\
\text { sectional } \\
\text { study }\end{array}$ & 32 & - & $\begin{array}{l}\text { To assess the } \\
\text { relationship } \\
\text { between } \\
\text { microbiota } \\
\text { composition and } \\
\text { clinical stages of } \\
\text { breast cancer. }\end{array}$ & $\begin{array}{l}\text { The absolute number of gut bacteria differed } \\
\text { according to clinical grades and BMI. Patients } \\
\text { with grade } 3 \text { breast cancer had relatively more } \\
\text { absolute numbers of Blautia spp. }\end{array}$ & $\begin{array}{l}\text { Gut microbiota } \\
\text { composition differs } \\
\text { according to clinical } \\
\text { grades of breast cancer } \\
\text { and BMI. }\end{array}$ \\
\hline $\begin{array}{l}\text { Fuhrman } \\
\text { BJ et al. } \\
\text { [32] }\end{array}$ & 2014 & $\begin{array}{l}\text { Observational: } \\
\text { Cross- } \\
\text { sectional } \\
\text { study. }\end{array}$ & 60 & $55-69$ years & $\begin{array}{l}\text { To investigate } \\
\text { whether urinary } \\
\text { estrogens and } \\
\text { estrogen } \\
\text { metabolite } \\
\text { levels are } \\
\text { associated with } \\
\text { the diversity and } \\
\text { composition of } \\
\text { the fecal } \\
\text { microbiome. }\end{array}$ & $\begin{array}{l}\text { The ratio of metabolites to parents was directly } \\
\text { associated with phylogenetic diversity }(\mathrm{R}=0.35 \text {, } \\
\mathrm{P}=.01) \text { and relative abundances of the order } \\
\text { Clostridiales }(\mathrm{R}=0.32, \mathrm{P}=.02) \text { and the } \\
\text { genus Bacteroides }(\mathrm{R}=-0.30, \mathrm{P}=.03) \text {. }\end{array}$ & $\begin{array}{l}\text { Women with higher gut } \\
\text { microbiome diversity had } \\
\text { an increased urinary ratio } \\
\text { of hydroxylated estrogen } \\
\text { metabolites to parent } \\
\text { estrogens. }\end{array}$ \\
\hline $\begin{array}{l}\text { Flores et } \\
\text { al. [33] }\end{array}$ & 2012 & $\begin{array}{l}\text { Observational } \\
\text { study: Cross- } \\
\text { sectional } \\
\text { study }\end{array}$ & 51 & $\begin{array}{l}25 \text { men, seven } \\
\text { postmenopausal } \\
\text { women, and } 19 \\
\text { premenopausal } \\
\text { women }\end{array}$ & $\begin{array}{l}\text { members and } \\
\text { functions of the } \\
\text { intestinal } \\
\text { microbiota that } \\
\text { influence } \\
\text { estrogen levels } \\
\text { via } \\
\text { enterohepatic } \\
\text { recirculation. }\end{array}$ & $\begin{array}{l}\text { Urinary estrogen levels in men and } \\
\text { postmenopausal women correlated directly with } \\
\text { fecal microbiome richness and alpha diversity } \\
\text { ( } R \geq 0.50, P \leq 0.003 \text { ) and fecal Clostridia taxa. In } \\
\text { addition, fecal } \beta \text {-glucuronidase correlated } \\
\text { proportionally with estrone and inversely with } \\
\text { total fecal estrogens ( } R \leq-0.47, P \leq 0.01) \text {. However, } \\
\text { in premenopausal women, these associations } \\
\text { were absent }(P \geq 0.6) \text {. }\end{array}$ & $\begin{array}{l}\text { Levels of non-ovarian } \\
\text { estrogens are associated } \\
\text { with gut microbial } \\
\text { richness and diversity. } \\
\text { This likely affects the risk } \\
\text { for estrogen-related } \\
\text { conditions in older adults } \\
\text { but not in premenopausal } \\
\text { women. }\end{array}$ \\
\hline $\begin{array}{l}\text { Zhu et } \\
\text { al. [34] }\end{array}$ & 2018 & $\begin{array}{l}\text { Observational: } \\
\text { Case-control } \\
\text { study }\end{array}$ & 133 & $\begin{array}{l}\text { Premenopausal } \\
\text { cases and } \\
\text { controls, } \\
\text { postmenopausal } \\
\text { cases and } \\
\text { postmenopausal } \\
\text { controls }\end{array}$ & $\begin{array}{l}\text { To evaluate the } \\
\text { composition and } \\
\text { functional } \\
\text { capacity of gut } \\
\text { microbiota of } \\
\text { breast cancer } \\
\text { patients } \\
\text { compared to } \\
\text { controls. }\end{array}$ & $\begin{array}{l}\text { Relative abundance of gut microbiota species } \\
\text { differed significantly, and microbial diversity was } \\
\text { higher in postmenopausal breast cancer cases } \\
\text { and controls but did not differ between } \\
\text { premenopausal cases and controls. The gut } \\
\text { metagenomes of postmenopausal breast cancer } \\
\text { patients had many genes encoding } \\
\text { lipopolysaccharide biosynthesis, iron complex } \\
\text { transport system, secretion system, and beta- } \\
\text { oxidation. }\end{array}$ & $\begin{array}{l}\text { There were notable } \\
\text { differences in the gut } \\
\text { microbial composition } \\
\text { and activity between } \\
\text { postmenopausal breast } \\
\text { cancer patients and } \\
\text { healthy controls. The gut } \\
\text { microbiota also } \\
\text { influences host immunity } \\
\text { and metabolic balance. }\end{array}$ \\
\hline $\begin{array}{l}\text { Goedert } \\
\text { et al. } \\
\text { [35] }\end{array}$ & 2015 & $\begin{array}{l}\text { Observational: } \\
\text { Case-control } \\
\text { Pilot study }\end{array}$ & 96 & $\begin{array}{l}\text { Postmenopausal } \\
\text { women }\end{array}$ & $\begin{array}{l}\text { do seek } \\
\text { differences } \\
\text { between the gut } \\
\text { microbiota in } 48 \\
\text { postmenopausal } \\
\text { breast cancer } \\
\text { cases and } 48 \\
\text { controls. }\end{array}$ & $\begin{array}{l}\text { Cases had altered microbiota composition ( } \beta \text { - } \\
\text { diversity, } \mathrm{P}=.006) \text { and lower a-diversity }(\mathrm{P}=.004) \\
\text { compared to controls. }\end{array}$ & $\begin{array}{l}\text { Postmenopausal women } \\
\text { with breast cancer have } \\
\text { altered composition and } \\
\text { estrogen-independent } \\
\text { low diversity of their gut } \\
\text { microbiota. }\end{array}$ \\
\hline
\end{tabular}

TABLE 2: Observational studies showing the association between gut microbiota diversity, estrogen levels, and breast cancer.

Obesity: Gut microbial dysbiosis can lead to obesity [37]. The concept of microbe-induced obesity was proposed as a condition where increased adiposity occurs due to primary disruption of the microbiota in early life by various factors such as antibiotics [37]. Obesity is a known breast cancer risk factor mostly via its effect on estrogen levels. Increased adiposity increases the risk of breast cancer through peripheral aromatization of androgens and suppression of production of hepatic hormone-binding proteins resulting in elevated free and total estrogens [11]. A relationship between cancer risk and adult weight gain was described where each 5-kilogram increase in weight was associated with an additional $11 \%$ increase in the risk of postmenopausal breast cancer [37]. Therefore, modulation of the gut microbiota to restore 
normobiosis can be a targeted approach against obesity and its associated breast cancer risk. Administration of soy isoflavones to postmenopausal women has been demonstrated to suppress Clostridiaceae, a family of Clostridiae that has been linked to obesity [10]. This would reduce the risk of breast cancer associated with obesity.

Non-Estrogen-Dependent Effects of the Gut Microbiota

Gut bacteria can play a protective role by producing cancer-protective metabolites. They also have an impact on immune regulation and DNA integrity.

Metabolism of bile acids: Bile acids found in the breast tissue originally come from the gut [23]. Lithocholic acid (LCA) is a bile acid produced only by intestinal bacteria such as Clostridia spp. from primary bile acids [23]. It has been found to exert antitumor effects with a 10-20\% decrease in breast cancer cell proliferation and inhibition of epithelial to mesenchymal cell transition $[23,38]$. It has antitumor immunity in murine models and in vitro, acting via the TGR5 receptor [23,38]. It also increases p53 expression, hence reducing cell death, and inhibits vascular endothelial growth factor (VEGF) production [23-24,38]. LCA causes changes in cellular metabolism by inducing glycolysis, the tricarboxylic acid (TCA) cycle, and mitochondrial oxidative phosphorylation in breast cancer cells that depend on Warburg metabolism $[24,36]$. Lithocholic acid (LCA) levels and levels of the LCA-producing enzyme gene were found to be low in fecal DNA of cases with early breast cancer relative to controls indicating reduced biosynthesis by gut microbiota in early breast cancer [36]. This shows the correlation between the composition of gut microbiota, lithocholic acid production, and its antiproliferative effects on breast cancer, which presents a potential target for intervention.

Production of short-chain fatty acids: Short-chain fatty acids (SCFAs), mainly butyrate, propionate, acetate, and lactate, are generated from the fermentation of indigestible carbohydrates by the intestinal microbiome and a small proportion by degradation of branched-chain amino acids [23]. The most protective SCFA is butyrate, which has the anticancer activity of inducing cancer cell apoptosis via mitochondrial reactive oxygen species (ROS) generation, anti-inflammatory effects, and inhibition of histone deacetylation via the Warburg effect [10,23,38]. Butyrate also suppresses angiogenesis, inhibiting tumor formation [38]. Its concentration in the intestine is dependent on diet and gut microbiota composition [38]. A high-fiber diet promotes butyrogenesis and proliferation of butyrate-producing microbes and, hence, is considered cancerprotective. Important butyrate-producing microbes include Faecalibacterium prausnitzii, Roseburia intestinalis, and Eubacterium rectale [38]. Reduction in the proportion of butyrate-producing bacteria could increase tumorigenesis and inflammation [10]. Therefore, butyrate is a potential anticancer metabolite whose production can be facilitated by dietary changes that modulate the gut microbiome. This can be applied in breast cancer prevention and treatment adjunctively.

Amino acid degradation: Metabolism of dietary amino acids produces biogenic amines with various functions. Cadaverine, a biogenic amine, is preferentially synthesized by bacterial enzymes from the decarboxylation of lysine [23]. Bacteria with the ability to produce cadaverine include members of the genera Enterococcus, Enterobacter, Escherichia, Proteus, Streptococci, and Shigella [24,38]. Cadaverine supplementation in vitro was found to inhibit breast cancer cells growth by inhibiting cellular migration, invasion, metastasis, and suppressing epithelial to mesenchymal transition [23,38]. It acts via trace amino acid receptors (TAAR) on target cells $[24,38]$. Its levels were found to be reduced in patients with early-stage breast cancer, possibly due to decreased microbial production demonstrating its role in the reduction of breast cancer risk and progression [39].

DNA damage: Intestinal microbes can trigger malignant transformation by disrupting genomic stability, causing resistance to cell death, and altering cell proliferation [26]. In addition, gut microbiota can cause damage to the host DNA by causing carcinogenic mutations and by releasing toxins into host cells, for example, from Bacillus fragilis, which can cause carcinogenic cell responses [26]. Increased reactive oxygen species production induced by some bacteria also damages DNA [26].

Immune regulation: Microbes can facilitate malignancy by stimulation of an unregulated inflammatory immune response [26]. Dysbiosis results in shifts in the bacterial metabolites toward an inflammatory state that favors and propagates carcinogenesis [23]. Gut bacteria were found to require neutrophils to promote tumorigenesis in distant sites such as the mammary gland in mice, demonstrating the involvement of immune cells in tumor formation [40]. An intact intestinal barrier is an essential component of the immunity that prevents translocation of gut bacteria to distant sites such as the breast and the release of lipopolysaccharide (LPS) from gut bacteria into the blood [38]. A healthy gut barrier involves the maintenance of tight junctions between epithelial cells by proteins such as claudins and the presence of a thick mucus lining maintained by some bacteria [38]. A state of dysbiosis compromises the cellular adhesion proteins and reduces the bacterial species that maintain the thick mucus lining leading to increased barrier permeability, also referred to as a 'leaky gut' [38]. An imbalance between regulatory $\mathrm{T}$ cells and inflammation-associated Th17 cells (increased) also occurs, inciting both local and systemic inflammatory responses [38]. Increased circulating LPS has been associated with breast cancer metastasis via activation of monocyte-activated endothelial adhesion of circulating cancer cells [38]. On the other hand, 
Faecalibacterium prausnitzii was found to suppress the growth of breast cancer cells via its inhibitory action on the IL6/STAT3 pathway showing a potential avenue for intervention that should be explored further [41].

\section{Application to diagnosis, prevention, and treatment of breast cancer}

The intestinal microbiome can be useful in the diagnosis of breast cancer. The microbiota of breast cancer patients needs to be profiled to allow us to conclusively determine if a causative association exists between gut microbiota and breast cancer. This would identify the gut microbiota as an additional environmental risk factor and a potential prognostic modulator of the disease [42]. An ongoing case-control clinical trial aims to demonstrate the association between breast cancer and the mammary and intestinal microbiota characteristics by undertaking simultaneous analysis of both [42]. It also attempts to link the microbiota with endocrine disruption and the risk of breast cancer [42]. Investigating variations in composition and functionality of the estrobolome in healthy individuals relative to estrogen-driven breast cancer patients can lead to the development of microbiome-based biomarkers and future targeted interventions to reduce the risk of breast cancer [11]. Correlations to specific bacterial taxa associated with breast cancer, such as Clostridia should be carefully explored as part of potential cancer detection strategies [33]. Ma et al. found that Faecalibacterium abundance was reduced in breast cancer patients and had a negative correlation with phosphorocholine [36]. This implies that combining the detection of flora bacteria such as Faecalibacterium and flora metabolites such as phosphorolcholine could be useful in breast cancer detection [36].

Disruption of the gut microbiome results in variations in the microbiome-derived cancer-protective metabolites, promoting carcinogenesis in distant organs, in this case, the breast. Thus, strategies to promote a gut microbiota profile that enables the production of cancer-protective bacterial metabolites via targeted dietary or supplementary interventions in women with breast cancer could improve their health outcomes. Although precise mechanisms remain undetermined, this is an up-and-coming area to explore.

Interventional clinical studies should be designed with the aim of restoring the gut dysbiosis found in breast cancer patients toward a composition similar to that of healthy women [42]. Prebiotics and probiotics can be considered for use to prevent or arrest breast cancer development by restoring normobiosis [10]. Their active compounds can be purified and used as adjunctive therapy. Soy isoflavones such as daidzein are prebiotics from a class of phytoestrogens that are metabolized by bacteria to a biologically active metabolite called equol that has been shown to have defensive activity against breast cancer, among other diseases [43]. However, only about $30-50 \%$ of people can convert isoflavones to equol, probably due to variations in microbiota composition. In this regard, investigation needs to be done on improving the production of equol, perhaps by administering daidzein with probiotic bacteria as a potential preventative strategy against breast cancer in menopausal women [43]. Dietary fiber is a well-known form of a prebiotic that is fermented by gut bacteria producing cancer-protective metabolites such as butyrate [36]. There is an ongoing interventional clinical trial whose objective is to investigate the effect of a novel probiotic on the breast and gut microbiome in breast cancer as well as on the quality of life of breast cancer patients [44]. Herbal supplements such as American ginseng can produce cancer-protective metabolites and regulate intestinal microbiota composition to prevent tumorigenesis [10]. The spores of Ganoderma lucidum have generated interest due to their anti-inflammatory and anticancer properties that result from restoring exhausted $\mathrm{T}$ cells by increasing cytotoxic $\mathrm{T}$ cell population and downregulating the suppressive co-inhibitors; programmed cell death protein (Pd-1) and cytotoxic T lymphocyte antigen-4 (CTLA-4) [45]. Ganoderma lucidum spores could also be used to modulate the intestinal microbiota and restore normobiosis [45]. Conclusive clinical trials need to be done to demonstrate the benefits of incorporating these substances to prevent and treat breast cancer.

\section{Conclusions}

The burden of breast cancer still looms over our heads, and we ought to stay ahead of the curve. Gut microbial dysbiosis seemingly has a role to play in the development of some cases of breast cancer and has untapped life-saving potential as a target for intervention. Several factors disrupt normobiosis in the gut and homeostasis, favoring the development of disease. The gut bacteria influence estrogen metabolism and adiposity, which are both associated with breast cancer. The microbiota are also involved in producing cancer-protective metabolites such as butyrate, cadaverine, and lithocholic acid. Immune interactions with gut microbiota and microbial effects on host DNA also play a role in the development of breast cancer and are potential targets of treatment. The evidence of all these correlations marks the gut microbiome as a hub for further exploration into novel approaches in diagnosis and treatment. Once well characterized, the gut microbiota profile and its metabolites may serve as biomarkers for breast cancer. Prebiotics, probiotics, and supplements should be explored for use in the prevention and adjunctive treatment of breast cancer by reversal of gut microbial dysbiosis. A few animal and in-vitro studies were incorporated in this review. We were limited by the paucity of human interventional clinical trials to validate the suggested treatment approaches and incomplete profiling of the intestinal microbiome of breast cancer patients making it difficult to ascertain a causal relationship between gut microbiota dysbiosis and breast cancer. Interventional clinical trials, where safe, are required to substantiate the use of the prevention and treatment strategies mentioned above. Large-scale profiling of the gut microbiome and the associated metabolome of breast cancer patients relative to that of healthy individuals will be beneficial to allow the determination of new biomarkers for the disease. 


\section{Additional Information \\ Disclosures}

Conflicts of interest: In compliance with the ICMJE uniform disclosure form, all authors declare the following: Payment/services info: All authors have declared that no financial support was received from any organization for the submitted work. Financial relationships: All authors have declared that they have no financial relationships at present or within the previous three years with any organizations that might have an interest in the submitted work. Other relationships: All authors have declared that there are no other relationships or activities that could appear to have influenced the submitted work.

\section{References}

1. Sung H, Ferlay J, Siegel RL, Laversanne M, Soerjomataram I, Jemal A, Bray F: Global Cancer Statistics 2020: GLOBOCAN estimates of incidence and mortality worldwide for 36 cancers in 185 countries. CA Cancer J Clin. 2021, 71:209-49. 10.3322/caac.21660

2. Tao Z, Shi A, Lu C, Song T, Zhang Z, Zhao J: Breast cancer: epidemiology and etiology. Cell Biochem Biophys. 2015, 72:333-8. 10.1007/s12013-014-0459-6

3. Siegel RL, Miller KD, Fuchs HE, Jemal A: Cancer statistics, 2021. CA Cancer J Clin. 2021, 71:7-33. 10.3322/caac. 21654

4. American Cancer Society: breast cancer risk and prevention . (2021). Accessed: June 10, 2021: https://www.cancer.org/cancer/breast-cancer/risk-and-prevention.html.

5. Breastcancer.Org: breast cancer risk factors . (2021). Accessed: June 10, 2021: https://www.breastcancer.org/risk/factors.

6. Nelson HD, Zakher B, Cantor A, et al.: Risk factors for breast cancer for women aged 40 to 49 years: a systematic review and meta-analysis. Ann Intern Med. 2012, 156:635-48. 10.7326/0003-4819-156-9201205010-00006

7. Sirisinha S: The potential impact of gut microbiota on your health: current status and future challenges . Asian Pac J Allergy Immunol. 2016, 34:249-64. 10.12932/AP0803

8. Fernández MF, Reina-Pérez I, Astorga JM, Rodríguez-Carrillo A, Plaza-Díaz J, Fontana L: Breast cancer and its relationship with the microbiota. Int J Environ Res Public Health. 2018, 15:1747. 10.3390/ijerph15081747

9. Yang J, Tan Q, Fu Q, et al.: Gastrointestinal microbiome and breast cancer: correlations, mechanisms and potential clinical implications. Breast Cancer. 2017, 24:220-8. 10.1007/s12282-016-0734-Z

10. Rea D, Coppola G, Palma G, et al.: Microbiota effects on cancer: from risks to therapies . Oncotarget. 2018, 9:17915-27. 10.18632/oncotarget.24681

11. Newman TM, Vitolins MZ, Cook KL: From the table to the tumor: the role of Mediterranean and western dietary patterns in shifting microbial-mediated signaling to impact breast cancer risk. Nutrients. 2019, 11:2565. 10.3390/nu11112565

12. Kwa M, Plottel CS, Blaser MJ, Adams S: The intestinal microbiome and estrogen receptor-positive female breast cancer. J Natl Cancer Inst. 2016, 108:djw029. 10.1093/jnci/djw029

13. Gorbach SL, Goldin BR: Diet and the excretion and enterohepatic cycling of estrogens . Prev medicine. 1987, 16:525-31. 10.1016/0091-7435(87)90067-3

14. Goldin BR, Adlercreutz H, Gorbach SL, Warram JH, Dwyer JT, Swenson L, Woods MN: Estrogen excretion patterns and plasma levels in vegetarian and omnivorous women. N Engl J Med. 1982, 307:1542-7. 10.1056/NEJM198212163072502

15. Adlercreutz H, Mousavi Y, Höckerstedt K: Diet and breast cancer. Acta Oncol. 1992, 31:175-81. 10.3109/02841869209088899

16. Friedman GD, Oestreicher N, Chan J, Quesenberry CP Jr, Udaltsova N, Habel LA: Antibiotics and risk of breast cancer: up to 9 years of follow-up of 2.1 million women. Cancer Epidemiol Biomarkers Prev. 2006, 15:2102-6. 10.1158/1055-9965.EPI-06-0401

17. Boursi B, Mamtani R, Haynes K, Yang YX: Recurrent antibiotic exposure may promote cancer formation-another step in understanding the role of the human microbiota?. Eur J Cancer. 2015, 51:2655-64. 10.1016/j.ejca.2015.08.015

18. Velicer CM, Heckbert SR, Lampe JW, Potter JD, Robertson CA, Taplin SH: Antibiotic use in relation to the risk of breast cancer. JAMA. 2004, 291:827-35. 10.1001/jama.291.7.827

19. Sørensen HT, Skriver MV, Friis S, McLaughlin JK, Blot WJ, Baron JA: Use of antibiotics and risk of breast cancer: a population-based case-control study. Br J Cancer. 2005, 92:594-6. 10.1038/sj.bjc.6602313

20. García Rodríguez LA, González-Pérez A: Use of antibiotics and risk of breast cancer . Am J Epidemiol. 2005, 161:616-9. 10.1093/aje/kwi087

21. Goldin BR, Swenson L, Dwyer J, Sexton M, Gorbach SL: Effect of diet and Lactobacillus acidophilus supplements on human fecal bacterial enzymes. J Natl Cancer Inst. 1980, 64:255-61. 10.1093/jnci/64.2.255

22. Lê MG, Moulton LH, Hill C, Kramar A: Consumption of dairy produce and alcohol in a case-control study of breast cancer. J Natl Cancer Inst. 1986, 77:633-6. 10.1093/jnci/77.3.633

23. Mikó E, Kovács T, Sebő É, et al.: Microbiome-microbial metabolome-cancer cell interactions in breast cancer-familiar, but unexplored. Cells. 2019, 8:293. 10.3390/cells8040293

24. Parida S, Sharma D: Microbial alterations and risk factors of breast cancer: connections and mechanistic insights. Cells. 2020, 9:1091. 10.3390/cells9051091

25. Komorowski AS, Pezo RC: Untapped "-omics": the microbial metagenome, estrobolome, and their influence on the development of breast cancer and response to treatment. Breast Cancer Res Treat. 2020, 179:287300. 10.1007/s10549-019-05472-w

26. Alizadehmohajer N, Shojaeifar S, Nedaeinia R, et al.: Association between the microbiota and women's cancers - Cause or consequences?. Biomed Pharmacother. 2020, 127:110203. 10.1016/j.biopha.2020.110203

27. Ervin SM, Li H, Lim L, Roberts LR, Liang X, Mani S, Redinbo MR: Gut microbial $\beta$-glucuronidases reactivate estrogens as components of the estrobolome that reactivate estrogens. J Biol Chem. 2019, 294:18586-99. 
10.1074/jbc.RA119.010950

28. Parida S, Sharma D: The microbiome-estrogen connection and breast cancer risk. Cells. 2019, 8:1642. 10.3390/cells8121642

29. Doisneau-Sixou SF, Sergio CM, Carroll JS, Hui R, Musgrove EA, Sutherland RL: Estrogen and antiestrogen regulation of cell cycle progression in breast cancer cells. Endocr Relat Cancer. 2003, 10:179-86. 10.1677/erc.0.0100179

30. Dabek M, McCrae SI, Stevens VI, Duncan SH, Louis P: Distribution of beta-glucosidase and betaglucuronidase activity and of beta-glucuronidase gene gus in human colonic bacteria. FEMS Microbiol Ecol. 2008, 66:487-95. 10.1111/j.1574-6941.2008.00520.x

31. Bard JM, Luu HT, Dravet F, et al.: Relationship between intestinal microbiota and clinical characteristics of patients with early stage breast cancer. FASEB J. 2015, 29:914.2. 10.1096/fasebj.29.1_supplement.914.2

32. Fuhrman BJ, Feigelson HS, Flores R, Gail MH, Xu X, Ravel J, Goedert JJ: Associations of the fecal microbiome with urinary estrogens and estrogen metabolites in postmenopausal women. J Clin Endocrinol Metab. 2014, 99:4632-40. 10.1210/jc.2014-2222

33. Flores R, Shi J, Fuhrman B, et al.: Fecal microbial determinants of fecal and systemic estrogens and estrogen metabolites: a cross-sectional study. J Transl Med. 2012, 10:253. 10.1186/1479-5876-10-253

34. Zhu J, Liao M, Yao Z, et al.: Breast cancer in postmenopausal women is associated with an altered gut metagenome. Microbiome. 2018, 6:136. 10.1186/s40168-018-0515-3

35. Goedert JJ, Jones G, Hua X, et al.: Investigation of the association between the fecal microbiota and breast cancer in postmenopausal women: a population-based case-control pilot study. J Natl Cancer Inst. 2015, 107:djv147. 10.1093/jnci/djv147

36. Mikó E, Vida A, Kovács T, et al.: Lithocholic acid, a bacterial metabolite reduces breast cancer cell proliferation and aggressiveness. Biochim Biophys Acta Bioenerg. 2018, 1859:958-74. 10.1016/j.bbabio.2018.04.002

37. Cox LM, Yamanishi S, Sohn J, et al.: Altering the intestinal microbiota during a critical developmental window has lasting metabolic consequences. Cell. 2014, 158:705-21. 10.1016/j.cell.2014.05.052

38. Sampsell K, Hao D, Reimer RA: The gut microbiota: a potential gateway to improved health outcomes in breast cancer treatment and survivorship. Int J Mol Sci. 2020, 21:9239. 10.3390/ijms21239239

39. Kovács T, Mikó E, Vida A, et al.: Cadaverine, a metabolite of the microbiome, reduces breast cancer aggressiveness through trace amino acid receptors. Sci Rep. 2019, 9:1300. 10.1038/s41598-018-37664-7

40. Lakritz JR, Poutahidis T, Mirabal S, et al.: Gut bacteria require neutrophils to promote mammary tumorigenesis. Oncotarget. 2015, 6:9387-96. 10.18632/oncotarget.3328

41. Ma J, Sun L, Liu Y, et al.: Alter between gut bacteria and blood metabolites and the anti-tumor effects of Faecalibacterium prausnitzii in breast cancer. BMC Microbiol. 2020, 20:82. 10.1186/s12866-020-01739-1

42. Plaza-Díaz J, Álvarez-Mercado AI, Ruiz-Marín CM, et al.: Association of breast and gut microbiota dysbiosis and the risk of breast cancer: a case-control clinical study. BMC Cancer. 2019, 19:495. 10.1186/s12885-0195660-y

43. Lopes DB, de Avila AR, de Queiros LD, Macedo JA, Macedo GA: Bioconversion of isoflavones into bioactive equol: state of the art. Recent Pat Food Nutr Agric. 2016, 8:91-8. 10.2174/2212798408666160620090519

44. Study to investigate efficacy of a novel probiotic on the bacteriome and mycobiome of breast cancer patients. (2021). Accessed: June 10, 2021: https://clinicaltrials.gov/ct2/show/NCT04362826.

45. Su J, Su L, Li D, et al.: Antitumor activity of extract from the sporoderm-breaking spore of Ganoderma lucidum: restoration on exhausted cytotoxic T cell With gut microbiota remodeling. Front Immunol. 2018, 9:1765. 10.3389/fimmu.2018.01765 